\title{
Gastrodin protects MC3T3-E1 osteoblasts from dexamethasone-induced cellular dysfunction and promotes bone formation via induction of the NRF2 signaling pathway
}

\author{
SHENGYE LIU ${ }^{1}$, TAO FANG ${ }^{1}$, LIYU YANG ${ }^{1}$, ZHIGUANG CHEN ${ }^{2}$, SHUAI MU ${ }^{1}$ and QIN FU $^{1}$ \\ ${ }^{1}$ Department of Spine and Joint Surgery and ${ }^{2}$ Emergency Department, The Shengjing Hospital of \\ China Medical University, Shenyang, Liaoning 110004, P.R. China
}

Received November 2, 2016; Accepted January 9, 2018

DOI: $10.3892 /$ ijmm.2018.3414

\begin{abstract}
Glucocorticoid (GC)-induced osteoporosis (GIO) is one of the most common secondary and iatrogenic forms of osteoporosis. GCs are widely used in clinical therapy and play a key role in the normal regulation of bone remodeling. However, the prolonged and high-dose administration of GCs results in the occurrence of osteoporosis, which is partially due to the dysfunction and apoptosis of osteoblasts and osteocytes. The aim of the present study was to investigate the effects of gastrodin, a natural bioactive compound isolated from the traditional Chinese herbal agent Gastrodia elata, on GC-treated MC3T3-E1 murine osteoblastic cells. MC3T3-E1 cells were exposed to dexamethasone (DEX), with or without gastrodin pretreatment, and cell viability was measured by the Cell Counting Kit-8 (CCK-8) assay. Quantitative polymerase chain reaction analysis was performed to evaluate osteogenic gene expression, and cellular alkaline phosphatase (ALP) activity was measured as well. Alizarin Red staining of calcium deposits was found to reflect the degree of osteoblast maturity. Western blotting was performed to determine the expression of osteogenic and adipogenic differentiation key proteins, as well as nuclear factor-like 2 (NRF2) pathway-related proteins. Annexin V-fluorescein isothiocyanate/propidium iodide flow cytometric analysis was performed to determine osteoblast apoptosis. JC-1 staining was used to detect the changes of the mitochondrial membrane potential in cells. The results revealed that gastrodin prevented the decrease in cell viability caused by DEX-induced MC3T3-E1 cell dysfunction, and that groups pretreated with gastrodin exhibited higher mRNA levels of osteogenic genes, such as Runx2, osterix, bone morphogenetic protein-2 and osteocalcin. Furthermore, treatment with both DEX and gastrodin was associated with increased ALP activity in MC3T3-E1 cells, as well as more calcium deposits, compared
\end{abstract}

Correspondence to: Dr Qin Fu, Department of Spine and Joint Surgery, The Shengjing Hospital of China Medical University, 36 Sanhao Street, Shenyang, Liaoning 110004, P.R. China

E-mail: CMU_FuQin@163.com

Key words: dexamethasone, gastrodin, glucocorticoid-induced osteoporosis, nuclear factor-like 2, osteoblast with cells treated with DEX alone. In addition, gastrodin increased osteogenic key marker protein Runx2 while activating NRF2 and downstream effector protein expression. Therefore, gastrodin may have the potential to reduce DEX-induced cell apoptosis and increase the mitochondrial membrane potential against DEX. These results demonstrated that gastrodin was able to prevent and/ or delay DEX-induced osteoporosis by improving osteoblast function, and these protective effects were verified in an animal model.

\section{Introduction}

Bone is a dynamic tissue with a relatively high turnover. The homeostasis of bone is tightly regulated by its constant formation and degradation to maintain its proper function and ensure adaptation to the load of the skeleton. Once this balance is disturbed, it may lead to bone sclerosis or osteoporosis. Osteoporosis is currently a major socioeconomic burden (1). Reduced bone content caused by various factors accelerates the degradation of bone microstructure and leads to bone brittleness. Bone density is reduced, leading to systemic skeletal symptoms, such as severe pain, multiple fractures, and potentially life-threatening events.

Osteoporosis may be classified as primary osteoporosis, which is observed in postmenopausal women, and secondary osteoporosis, which may be caused by several factors, such as malnutrition, endocrine problems, diabetes, genetics and medications, among which glucocorticoid (GC)-induced osteoporosis (GIO) is considered to be the most common secondary iatrogenic type of osteoporosis $(2,3)$. Even a daily dose as low as $2.5 \mathrm{mg}$ prednisone increases the risk of osteoporotic fractures, whereas inhaled GC therapy is also associated with bone loss. In extreme cases, a high dose may ultimately lead to systemic side effects, such as bone loss and osteoporotic osteonecrosis. It was previously reported that patients exhibited progressive loss of bone mass several weeks post-GC therapy. The patients' bone mass was found to decline rapidly within the first few months, reaching 5-15\% in total per year, and long-term GC therapy ( $>1$ year) was associated with an osteoporosis incidence rate is as high as $30-50 \%$ (4). Along with the occurrence of osteoporosis, osteoporotic fractures may occur, most commonly observed in the spinal vertebral body, proximal femur and ribs. Although the awareness of osteoporosis has increased in the general public, the severity of the side 
effects associated with the clinical use of GCs requires further elucidation; furthermore, the complex interplay between GCs and altered bone homeostasis is a subject of intense clinical interest. GC-induced osteogenic dysfunction of osteoblasts is considered to be one of the main pathological mechanisms underlying the development of GIO, including inhibition of osteoblast proliferation and osteogenic differentiation, or even induction of adipogenic differentiation and cell apoptosis (5). Natural medicines have been a subject of interest in recent years, as they are well known to exert diverse biological effects and improve bone quality. The aim of the present study was to investigate the therapeutic efficacy of gastrodin (GSTD), a natural medicinal extract, in reducing the adverse effects of high-dose dexamethasone (DEX) on MC3T3-E1 osteoblasts.

GSTD, also referred to as gastrodia glycosides, has been isolated from the traditional Chinese herbal agent Gastrodia elata and confirmed to be one of the major active components of Rhizoma gastrodiae. The molecular structure of GSTD is shown in Fig. 1, and its medicinal properties include smooth muscle-relaxing, anti-necrotic, anti-aging and anti-apoptotic effects $(6,7)$. GSTD effectively lowers lipid peroxidation levels, removes oxygen-free radicals, exerts antioxidant effects, reduces coupling oxidative phosphorylation and increases the levels of malondialdehyde and superoxide dismutase, as well as the expression levels of related genes and proteins $(8,9)$. Therefore, the main objective of the present study was to improve our knowledge of the therapeutic value of GSTD by examining its potential application as an inhibitor of GC-induced dysfunction of MC3T3-E1 cells. Furthermore, the possible molecular mechanisms underlying the effects of GSTD on MC3T3-E1 cell proliferation and differentiation under DEX treatment were investigated.

Regulatory transcription factor nuclear factor-like 2 (NRF2) is a transcription activator that can combine with antioxidant responsive element (ARE) and enhance the expression of antioxidant enzymes, including $\mathrm{NAD}(\mathrm{P}) \mathrm{H}$ :quinone oxidoreductase-1 (NQO-1) and heme oxygenase-1 (HO-1) (10). NRF2 acts as an important cell protective mechanism against exogenous or endogenous noxious stimuli (11), and its expression is closely correlated with cell differentiation (12). In recent years, there have been studies indicating that certain natural products, such as sulforaphane, and bioactive compounds referred to as avenanthramides, may block the cytotoxic effects of high-dose DEX through activation of the NRF2 pathway and osteogenic differentiation, as well as inhibition of the apoptosis-related caspase protein family in $\operatorname{GIO}(13,14)$. However, considering the safety profile and long history of these drugs or extracts, new pharmaceuticals must be developed and approved. It was previously reported that GSTD may activate NRF2 signaling in liver, nerve, and vascular smooth muscle cells, playing a key role in antioxidation and anti-inflammation. GSTD may also be used in human bone marrow mesenchymal stem cells to promote osteogenesis by reducing osteoclast differentiation $(13,15)$. GSTD may inhibit the key adipogenic differentiation factor peroxisome proliferator-activated receptor (PPAR) $\gamma$, reducing fat content in hypertensive model rats (16).

Bone formation largely depends on the proliferation and differentiation of osteoblasts and is required for skeletal development. It is well known that GCs suppress bone formation by attenuating osteoblast viability and differentiation (17). The

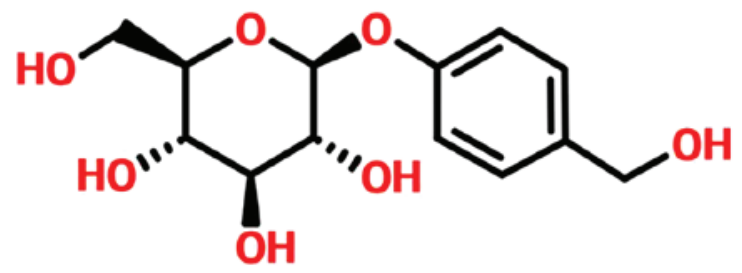

Figure 1. Chemical structure of gastrodin.

pathophysiology of GIO is complex, and GCs modify bone mass mainly by directly affecting osteoblasts (18). Whether GSTD can activate NRF2 and its downstream transcription factors to reduce the excessive stimulation induced by DEX in osteoblasts and reduce GC-induced osteoblast injury requires further investigation. Our results demonstrated that GSTD effectively promoted osteogenic differentiation in MC3T3-E1 cells through regulation of the NRF2 pathway. This study may provide a novel strategy for the prevention of GCs-induced osteoporosis by using natural products.

\section{Materials and methods}

Reagents. Purified GSTD (>98\%) was purchased from the National Institute for the Control of Pharmaceutical and Biological Products (Dalian, China), dissolved in dimethyl sulfoxide (DMSO; Sigma-Aldrich; Merck KGaA, St. Louis, MO, USA) and stored at $-20^{\circ} \mathrm{C}$. The final concentrations of GSTD were 0 (control), 1 and $5 \mu \mathrm{M}$, and the final concentration of DMSO in the culture was $<0.01 \%$. Minimum essential medium alpha modification ( $\alpha$-MEM), fetal bovine serum (FBS) and trypsin-EDTA were obtained from GE Healthcare Life Sciences HyClone Laboratories (Logan, UT, USA). The Cell Counting Kit-8 (CCK-8) was purchased from Sigma-Aldrich; Merck KGaA. The Annexin V-FITC apoptosis detection kit was obtained from Beyotime Institute of Biotechnology (Shanghai, China). Rabbit anti-HO-1 (cat. no. ab68477; 1:1,000), rabbit anti-NQO-1 (cat. no. ab76956; 1:1,000), and mouse anti- $\beta$ actin (cat. no. ab8245; 1:1,000), Anti-OCN (cat. no. ab93876; 1:1,000) monoclonal antibodies were purchased from Abcam (Cambridge, MA, USA). Rabbit anti-NRF2 (cat. no. 12721; 1:1,000), rabbit anti-Runx2 (cat. no. 20056S; 1:1,000), and rabbit anti-PPAR $\gamma$ (cat. no. 2430; 1:1,000) monoclonal antibodies were purchased from Cell Signaling Technology, Inc. (Danvers, MA, USA). TRIzol reagent was obtained from Thermo Fisher Scientific, Inc. (Carlsbad, CA, USA). Primers were designed and synthesized by Sangon Biotech Co., Ltd. (Shanghai, China).

Cell culture. MC3T3-E1 cells were maintained in $\alpha$-MEM (GE Healthcare Life Sciences HyClone Laboratories) supplemented with a $10 \% \mathrm{FBS}, 100 \mathrm{U} / \mathrm{ml}$ penicillin and $100 \mu \mathrm{g} / \mathrm{ml}$ streptomycin at $37^{\circ} \mathrm{C}$ with $5 \% \mathrm{CO}_{2}$ in a humidified atmosphere. Cells in the exponential phase of growth were selected for the experiments. For osteogenic differentiation, cells were cultured in inducing growth medium supplemented with $100 \mathrm{nM}$ DEX, $10 \mathrm{mM} \beta$-glycerophosphate and $50 \mathrm{mg} / \mathrm{ml}$ ascorbic acid (Sigma-Aldrich; Merck KGaA), replenished every 3 days.

Cell viability assay. Cell viability was assessed by the CCK-8 assay, which is used for detecting cell proliferation with a high 
sensitivity. Briefly, MC3T3-E1 cells were seeded at a density of $5 \times 10^{3}$ cells/well in 96-well plates and incubated in the growth media for $24 \mathrm{~h}$ at $37^{\circ} \mathrm{C}$. Subsequently, the cells were exposed to different concentrations of $\operatorname{DEX}(0,1,5,25,50,100$ and $200 \mu \mathrm{M})$ and $\operatorname{GSTD}(0,0.1,1,5,10,50$ and $100 \mu \mathrm{M})$ for $24 \mathrm{~h}$. Furthermore, the cells were also pretreated with GSTD $(1$ and $5 \mu \mathrm{M})$ for $2 \mathrm{~h}$, and then exposed to DEX $(200 \mu \mathrm{M})$ for $24 \mathrm{~h}$. Subsequently, $10 \mu \mathrm{l}$ CCK- 8 were added to each well and incubation was continued for $2 \mathrm{~h}$. The optical density was measured at $450 \mathrm{~nm}$ on a microplate reader with a microplate spectrophotometer (ELX800; BioTek, VT, USA) and the absorbance of each well was recorded. The ratio of the mean absorbance was considered to reflect the relative cellular viability.

Alkaline phosphatase (ALP) activity assay. To examine the ALP activity in MC3T3-E1 cells, the cells were seeded into 12 -well plates at a density of $5 \times 10^{4}$ cells/well in different drug-treated osteogenic-inducing media. The cells were washed twice, harvested and lysed with $100 \mu \mathrm{l}$ assay lysis buffer (Beyotime Institute of Biotechnology) after 7 days of culture. The ALP activity levels $(\mathrm{U} / \mathrm{ml})$ were measured with an ALP reagent kit (Nanjing Jiancheng Bioengineering Research Institute, Nanjing, China), according to the manufacturer's instructions. All samples were examined in triplicate.

Flow cytometric analysis of osteoblast apoptosis. MC3T3-E1 cells were incubated in 6-well plates at a density of $2 \times 10^{5}$ cells/well for $24 \mathrm{~h}$. The cells were then exposed to DEX in the presence or absence of different concentrations of GSTD for indicated time periods. The cells were harvested and resuspended in $300 \mu \mathrm{l}$ binding buffer containing $5 \mu \mathrm{l}$ Annexin V-FITC and $5 \mu \mathrm{l}$ propidium iodide (PI), then rinsed with phosphate-buffered saline (PBS). After washing twice with PBS, the cells were placed in an ice bath for $0.5 \mathrm{~h}$ and the samples were analyzed by FACScan flow cytometry.

Determination of mitochondrial outer membrane potential $(M O M P)$. The lipophilic cationic fluorescent dye 5,5',6,6'-terachloro-1,1',3,3'-tetraethylbenzimidazol-carbocyanine iodide (JC-1) was used to detect the changes in MOMP $(\Delta \psi \mathrm{m})$ as an indicator of mitochondrial function. Carbonyl cyanide 3-chlorophenylhydrazone (CCCP 1.0 mM, 2 h; Sigma-Aldrich; Merck $\mathrm{KGaA})$ was used as positive control. Cells $\left(2 \times 10^{5}\right.$ cells/well $)$ were cultured in 6-well plates. The groups were as previously designed, treated with different concentrations of GSTD and DEX for $24 \mathrm{~h}$. Measurements were performed using a MOMP detection kit (Beyotime Institute of Biotechnology) according to the manufacturer's instructions. In brief, the cells were collected and then incubated with the MOMP-sensitive fluorescent dye $\mathrm{JC}-1$ for $20 \mathrm{~min}$ at $37^{\circ} \mathrm{C}$, washed twice in PBS, and subjected to flow cytometry.

Mineralization assay. Preosteoblast MC3T3-E1 cells were cultured in complete growth medium in $35-\mathrm{mm}$ dishes. When the cells reached $80 \%$ confluence, mineralization was induced by culturing confluent cells in growth medium supplemented with $10 \mathrm{mM} \beta$-glycerophosphate and $50 \mathrm{mg} / \mathrm{ml}$ ascorbic acid (Sigma-Aldrich; Merck KGaA). Inducing media were replenished every 3 days. After 21 days, the cells were washed with PBS and fixed in 90\% ethanol (Sigma-Aldrich; Merck KGaA) at room temperature for $30 \mathrm{~min}$. After rinsing with distilled water, the cells were stained with $2 \mathrm{ml}$ of $10 \mathrm{mM}$ Alizarin Red S ( $\mathrm{pH} 4$.2) per dish at room temperature for 15 min while being gently swayed. Following aspiration of the unincorporated dye, the cells were washed four times with $2 \mathrm{ml}$ distilled water to exclude non-specific staining. Mineralized nodules and stained cells were visualized and photographed with a phase contrast microscope (Zeiss, Oberkochen, Germany).

Quantification of gene expression by quantitative polymerase chain reaction $(q P C R)$. Cells were cultured in osteogenic-inducing media with drug treatment for 2 days. Total RNA was extracted with TRIzol reagent, then used to synthesize cDNA using SuperScript II reverse transcriptase (Invitrogen; Thermo Fisher Scientific, Inc.) with $5 \mu$ g oligo(dT) primers per sample. By using SYBR-Green PCR master mix (Applied Biosystems; Thermo Fisher Scientific, Inc., Waltham, MA, USA), qPCR was performed in a total volume of $20 \mu \mathrm{l}$ in a 7500 Real-Time PCR system (Applied Biosystems; Thermo Fisher Scientific, Inc.) as follows: $95^{\circ} \mathrm{C}$ for $5 \mathrm{~min}$, followed by 40 cycles of $95^{\circ} \mathrm{C}$ for $30 \mathrm{sec}$ and $60^{\circ} \mathrm{C}$ for $45 \mathrm{sec}$. Melting curve analysis was used to confirm the specificity of the amplification and glyceraldehyde 3-phosphate dehydrogenase (GAPDH) served as the endogenous control for normalization of the amount of total RNA in each group. The relative levels of gene expression were calculated as follows: $\Delta \mathrm{Cq}=\mathrm{Cq}_{\text {gene }}-\mathrm{Cq}_{\text {reference }}$; gene expression was calculated as fold change according to the $2^{-\Delta \Delta \mathrm{Cq}}$ method and measurements were repeated independently in triplicate. The primer sequences were as follows: Forward, 5'-CTGACCACCTGAACTCCAC-3' and reverse, 5'-CATCTA GGTACAACATGGAG-3' for BMP-2; forward, 5'-GAATGC ACTACCCAGCCAC-3' and reverse, 5'-TGGCAGGTACGT GTGGTAG-3' for Runx2; forward, 5'-GTCAAGAGTCTT AGCCAAACTC-3' and reverse, 5'-AAATGATGTGAGGCC AGATGG-3' for osterix (OSX); forward, 5'-CAATAAGGT AGTGAACAGAC-3' and reverse, 5'-CTTCAAGCCATACTG GTCT-3' for osteocalcin (OCN); and forward, 5'-GTGAAG CAGGCATCTGAGGG-3' and reverse, 5'-GCCGTATTCATT GTCATACCAGG-3' for GAPDH.

Western blot analysis. Total protein from each well was harvested in ice-cold radioimmunoprecipitation (RIPA) lysis buffer (Thermo Fisher Scientific, Inc.) supplemented with phenylmethanesulfonyl fluoride for $1 \mathrm{~h}$. The protein concentration was quantified using the bicinchoninic acid protein assay kit (Sigma-Aldrich; Merck KGaA) according to the manufacturer's instructions. Equal proteins of each treatment were separated on $12 \%$ sodium dodecyl sulfate-polyacrylamide gel electrophoresis (Beyotime Institute of Biotechnology) and electrophoretically transferred onto polyvinylidene difluoride membranes (Millipore, Bedford, MA, USA). The membranes were soaked in 5\% skimmed milk as blocking buffer for $1 \mathrm{~h}$, then washed 3 times in Tris-buffered saline Tween-20 [TBST; $150 \mathrm{mM} \mathrm{NaCl}$ (pH 7.5), 20 mM Tris-HCl and $0.1 \%$ Tween-20] at room temperature. The membranes were incubated with primary monoclonal antibodies against Runx2, NRF2, NQO-1, HO-1, PPAR $\gamma$ and caspase-3 at 1:1,000 dilution overnight at $4^{\circ} \mathrm{C}$ followed by hybridization with horseradish peroxidase-conjugated secondary antibody (Santa Cruz Biotechnology, Inc., Santa Cruz, CA, USA) and visual- 
A

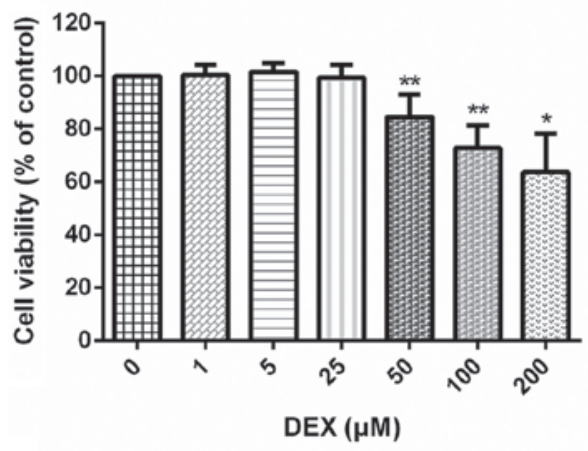

C

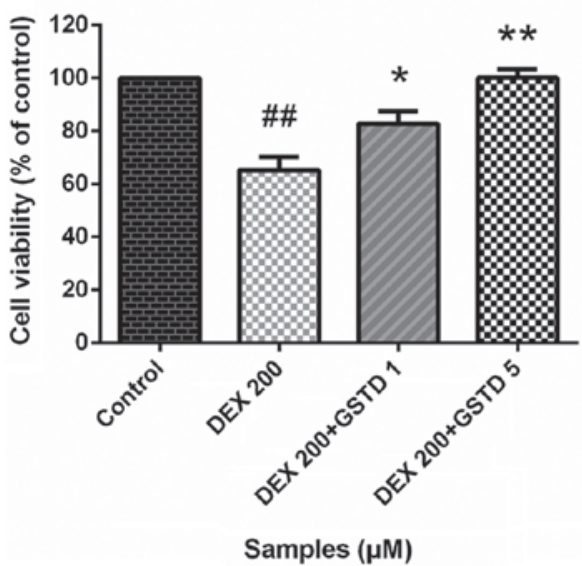

B

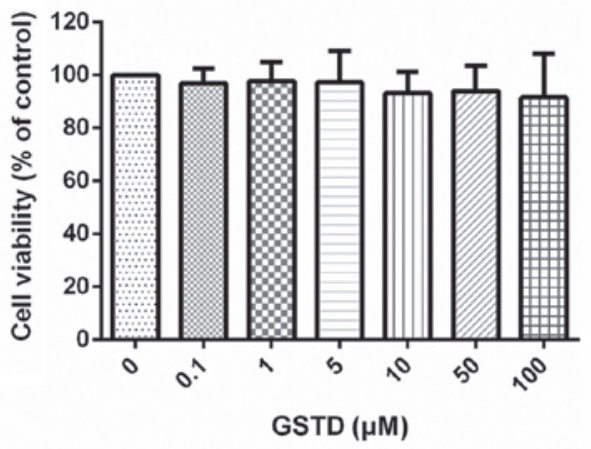

D

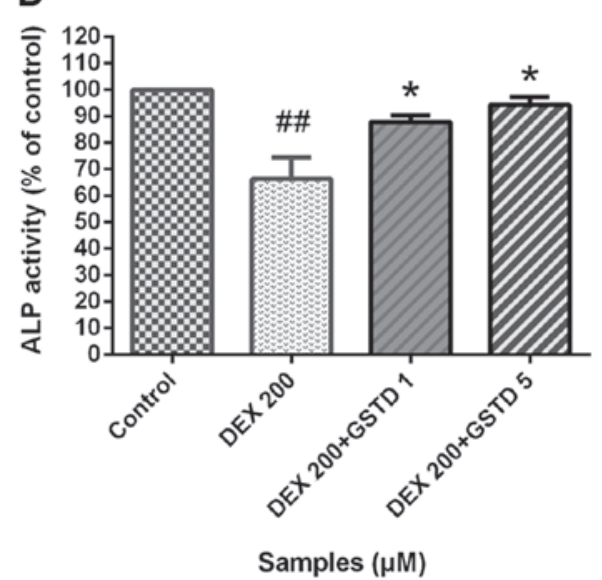

Figure 2. (A-C) Effects of gastrodin (GSTD) on the viability of MC3T3-E1 cells. The cells were incubated with different concentrations of (A) GSTD and (B) dexamethasone (DEX) alone or (C) simultaneously for $48 \mathrm{~h}$. Cell viability was determined by the Cell Counting Kit-8 (CCK-8) assay. The experiment was performed in triplicate and data are presented as the mean \pm standard deviation (SD). ${ }^{\# \#} \mathrm{P}<0.01$ vs. control; ${ }^{* *} \mathrm{P}<0.01$ and ${ }^{*} \mathrm{P}<0.05$ vs. DEX $200 \mu \mathrm{M}$. (D) Effect of GSTD and $200 \mu \mathrm{M}$ DEX on alkaline phosphatase (ALP) activity in MC3T3-E1 cells. Data are presented as the mean $\pm \mathrm{SD}$. ${ }^{\# \#} \mathrm{P}<0.01 \mathrm{vs}$. control; ${ }^{*} \mathrm{P}<0.05 \mathrm{vs}$. DEX $200 \mu \mathrm{M}$.

ized using enhanced chemiluminescence. The relative protein levels were calculated based on $\beta$-actin as the loading control.

Animals and drug supplementation. A total of 32 female experimental Sprague Dawley rats, aged 8 weeks and weighing $250 \pm 22 \mathrm{~g}$, were obtained from the Animal Center of Shengjing Hospital of China Medical University (Shenyang, China). The rats were acclimatized to specific pathogen-free laboratory conditions (a well-ventilated controlled room at $20^{\circ} \mathrm{C}$ on a $12-\mathrm{h}$ light/dark cycle with free access to water and food) for 1 week prior to the drug treatments. All animal care and experimental procedures were approved by the Institutional Animal Care Ethics and Use Committee of China Medical University, and performed in accordance with the guidelines. The rats were randomly divided into 4 groups: Control, DEX and DEX with GSTD ( 1 and $5 \mathrm{mg} / \mathrm{kg} /$ day) groups. A GIO rat model $(\mathrm{n}=8)$ was induced by intramuscularly injected DEX $(1 \mathrm{mg} / \mathrm{kg} /$ day DEX for 60 days), while the control group ( $n=8)$ was administered an equivalent volume of normal saline. The GSTD therapeutic groups $(n=16)$ received different doses of GSTD (1 and $5 \mathrm{mg} / \mathrm{kg} /$ day) by gavage simultaneously with induction of the model by DEX. All the procedures lasted for 60 days to ensure the experimental modeling of the animals until bone samples (bilateral femora) were removed for further analysis.

Histology and bone mineral density (BMD) measurement. Following completion of animal modeling, the left femora were removed and placed in $4 \%$ paraformaldehyde for $48 \mathrm{~h}$ prior to demineralization for 30 days in 10\% EDTA solution that was replenished every 2 days. The femora were then processed in paraffin and were serially cut sagittally into $5-\mu \mathrm{m}$ sections for hematoxylin and eosin (H\&E) staining (Beyotime Institute of Biotechnology). The microstructure photograph and analysis were conducted in the proximal metaphysis adjacent to the epiphyseal growth plate. The right femora were directly used for BMD measurement by dual X-ray absorptiometry using a PIXImus II densitometer (GE Medical Systems, Lunar Division, Madison, WI, USA) and data were recorded for further analysis. The measurements were limited to the proximal femoral area.

Statistical analysis. All the presented data and results were processed using GraphPad Prism 6.01 software and expressed as mean \pm standard deviation of at least three independent experiments. One-way analysis of variance was used to determine statistical significance. $\mathrm{P}<0.05$ or $\mathrm{P}<0.01$ were considered to indicate statistically significant differences.

\section{Results}

GSTD pretreatment maintains MC3T3-E1 cell viability and ALP activity while exposed to high-dose DEX. To determine whether GSTD exerted protective effects on MC3T3-E1 cells against DEX-induced reduced viability, the effects of GSTD and DEX or combined effect of both drugs on MC3T3-E1 cell 
A

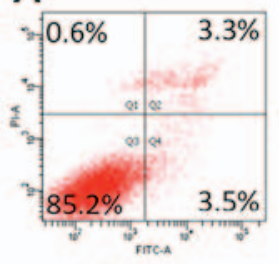

DEX $0 \mu \mathrm{M}$

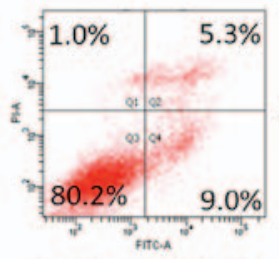

DEX $200 \mu \mathrm{M}+\mathrm{GSTD} 1 \mu \mathrm{M}$
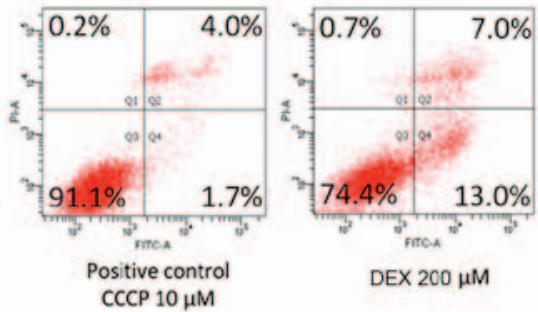

DEX $200 \mu \mathrm{M}$

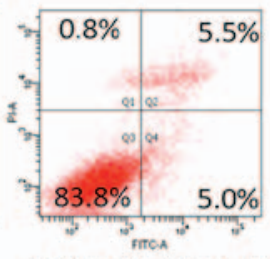

DEX $200 \mu \mathrm{M}+\mathrm{GSTD} 5 \mu \mathrm{M}$

B
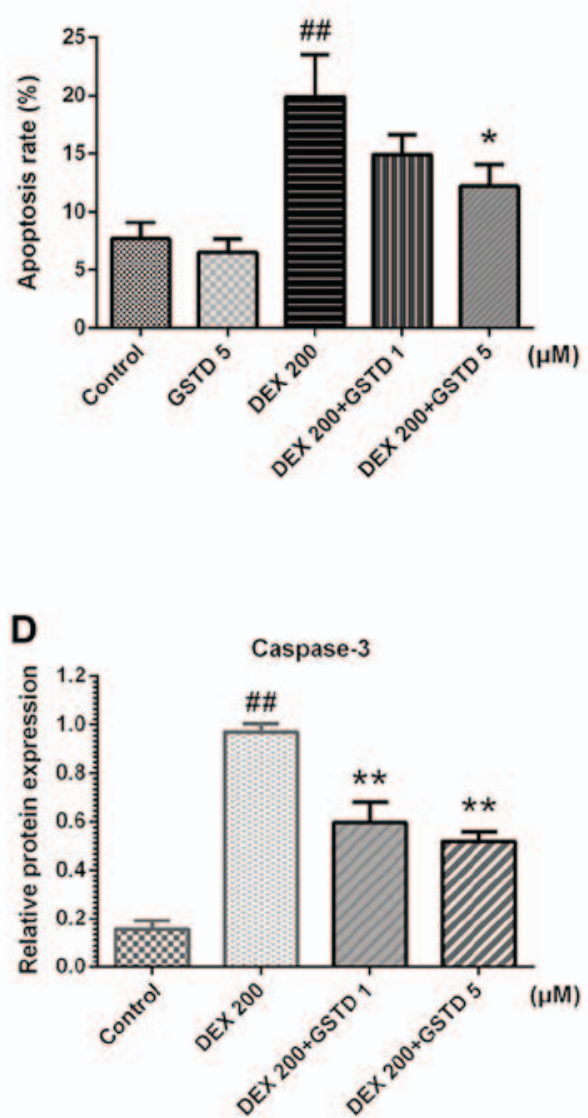

Figure 3. Effects of gastrodin (GSTD) on dexamethasone (DEX)-induced apoptosis in MC3T3-E1 cells, as observed following Annexin V-fluorescein isothianate/propidium iodide double staining and western blotting of apoptosis-related proteins. (A) The cells were treated with GSTD (1 and $5 \mu \mathrm{M}$ ) for $1 \mathrm{~h}$ prior to incubation with DEX $(200 \mu \mathrm{M})$ for $48 \mathrm{~h}$. Flow cytometric analysis was used to measure apoptotic rate. The upper left quadrant represents debris or dead cells, the lower left quadrant represents normal cells, while the upper and lower right quadrants represent late- and early-stage apoptotic cells, respectively. (B) MC3T3-E1 cell apoptotic rate statistically depicted in columns. Data are presented as mean \pm standard deviation (SD). (C and D) Western blotting and relative protein expression of caspase-3; data are expressed as mean $\pm \mathrm{SD}$. ${ }^{\# \#} \mathrm{P}<0.01$ and ${ }^{\#} \mathrm{P}<0.05$ vs. control; ${ }^{*} \mathrm{P}<0.05$ and ${ }^{* *} \mathrm{P}<0.01$ vs. $\mathrm{DEX}$.

viability were evaluated by the CCK-8 assay (Fig. 2). The CCK-8 assay indicated that GSTD affected the MC3T3-E1 cell viability marginally at concentrations of $\leq 100 \mu \mathrm{M}$ (Fig. 2B). Cell viability exhibited a noticeable decrease with DEX treatment at a concentration of $\geq 50 \mu \mathrm{M}$ (Fig. 2A), and $200 \mu \mathrm{M}$ was selected as the concentration in the DEX-induced decreased cell viability model, while GSTD at different concentrations (1 and $5 \mu \mathrm{M}$ ) was added to relieve this effect in a dose-dependent manner, indicating that GSTD was able to partially inhibit high-dose DEX-induced cytotoxicity and exerted a protective effect, although it did not entirely reverse the toxic effect.

ALP is a well-recognized indicator of osteoblast differentiation (19). ALP activity was examined to evaluate the extent of osteoblast differentiation. ALP activity exhibited significant differences between treatment with DEX and GSTD, alone or combined (Fig. 2D). As expected, DEX treatment significantly decreased ALP activity, while GSTD at 1 or $5 \mu \mathrm{M}$ was found to increase ALP activity against DEX by $~ 30 \%$; these findings indicate that GSTD also enhanced the activity of ALP compared with the DEX-treated group, suggesting that GSTD stimulated osteoblast differentiation, even under the inhibitory influence of DEX.
GSTD protects MC3T3-E1 cells from apoptosis. Annexin V-FITC/PI flow cytometric analysis was performed to determine cell apoptosis (Fig. 3). According to the results, DEX treatment markedly induced cell apoptosis, as previously reported (20), which was validated with a concentration of $200 \mu \mathrm{M}$ DEX, while combined pretreatment with GSTD significantly attenuated this effect in a dose-dependent manner and promoted cell survival. Moreover, the analysis also confirmed that GSTD per se improved cell survival, without any external stimulation, in a dose-dependent manner.

GSTD alleviated DEX-induced MOMP loss in MC3T3-E1 cells. JC-1 is a type of double-fluorescent dye, which has a good permeability through cellular and mitochondrial membranes. Once mitochondria are damaged, the mitochondrial membrane pores are opened, resulting in the loss of MOMP. The dye fluoresces red when it accumulates in healthy mitochondrial matrix to form J-aggregates with high membrane potential, whereas it fluoresces green as its monomeric form in affected mitochondria with diminished membrane potential. A decrease in the red/green fluorescence ratio manifests mitochondrial depolarization. CCCP is an inhibitor of the mitochondrial electron transport 
A
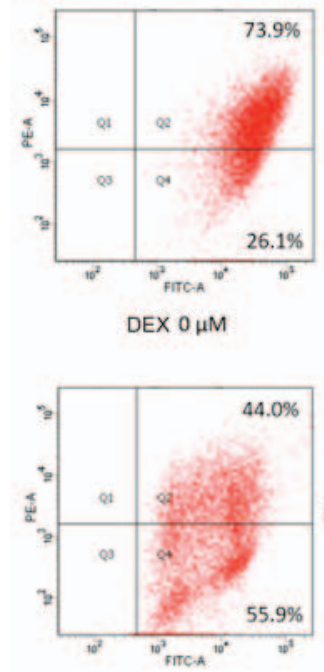

DEX $200 \mu \mathrm{M}+\mathrm{GSTD} 1 \mu \mathrm{M}$

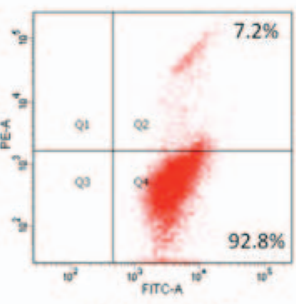

Positive control CCCP $10 \mu \mathrm{M}$

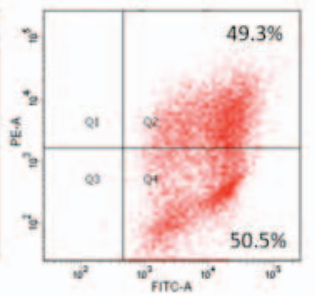

DEX $200 \mu \mathrm{M}+\mathrm{GSTD} 5 \mu \mathrm{M}$
B
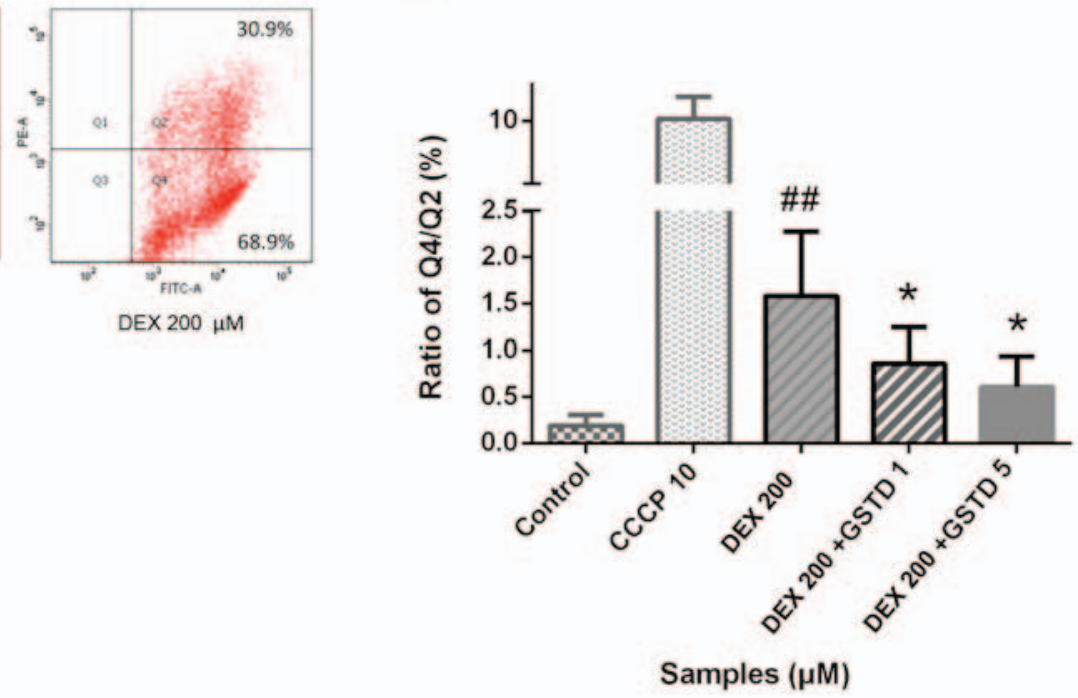

Figure 4. Gastrodin (GSTD) improved mitochondrial function by increasing mitochondrial outer membrane potential in dexamethasone (DEX)-treated MC3T3-E1 cells, as observed following JC-1 double-fluorescent dye staining. (A) The cells were pretreated with GSTD ( 1 and $5 \mu \mathrm{M})$ for $1 \mathrm{~h}$ prior to incubation with DEX (200 $\mu \mathrm{M})$ for $48 \mathrm{~h}$. On flow cytometric analysis, the upper right quadrant represents double-fluorescent dyed cells and the lower right quadrant represents single-fluorescent dyed cells. (B) The Q4/Q2 ratio was statistically analyzed. Data are presented as mean \pm standard deviation. ${ }^{\# \#} \mathrm{P}<0.01$ and ${ }^{\#} \mathrm{P}<0.05$ vs. control; ${ }^{*} \mathrm{P}<0.05$ and ${ }^{* *} \mathrm{P}<0.01 \mathrm{vs}$. DEX.

A
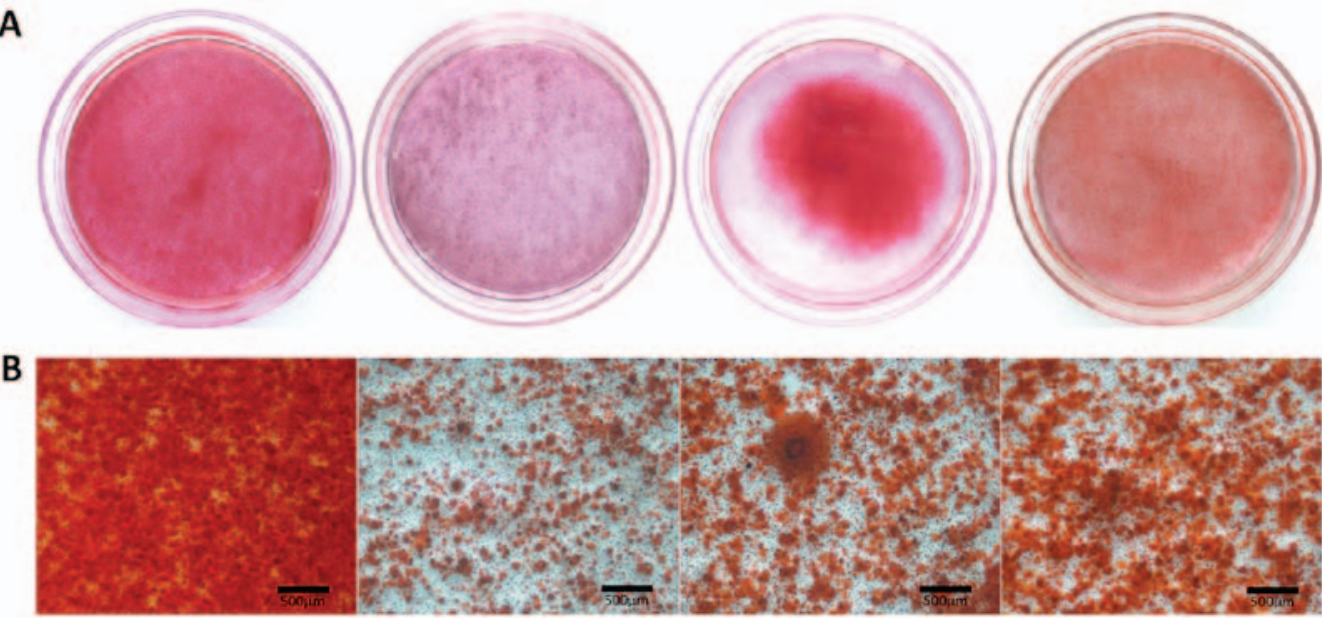

Control

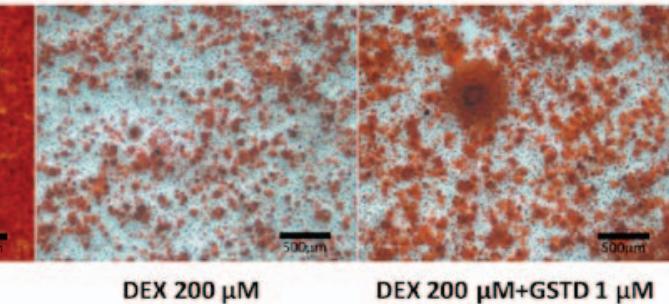

DEX $200 \mu \mathrm{M}+\mathrm{GSTD} 1 \mu \mathrm{M}$

Figure 5. Effect of gastrodin (GSTD) and dexamethasone (DEX) on osteogenic differentiation and mineralization. The mineralized matrix of MC3T3-E1 cells was stained with Alizarin Red S in 35-mm dishes following incubation with osteogenic medium for 21 days. (A) Visual inspection and (B) microscopic examination of the samples.

chain used as positive control, which may exert a toxic effect by decreasing MOMP. As shown in Fig. 4, GSTD increased MOMP against DEX treatment in a dose-dependent manner.

GSTD modulates DEX-induced matrix mineralization. Osteoblast osteogenic differentiation during the process of maturation is accompanied by mineralized bone nodule formation. Alizarin Red staining dye conjugates with mineralized extracellular matrix to form calcified deposits in the cells. The effect of GSTD on mineralization in cells was determined by Alizarin Red staining and microscopic examination. Results reported by Huang et al indicated that a reduction in reactive oxygen species by GSTD in human bone marrow mesenchymal stem cells (hBMMSCs) exerted a good recovery effect on calcium mineralization, suggesting that GSTD attenuated $\mathrm{H}_{2} \mathrm{O}_{2}$-induced hBMMSC dysfunction, demonstrating its protective effects on osteoblastogenic differentiation and calcium deposition (6). In the present study, the addition of DEX led to inhibition of calcified deposit formation, while GSTD significantly enhanced bone nodule formation and preserved calcification of MC3T3-E1 cells against high-dose DEX in a dose-dependent manner compared with that in the control group (Fig. 5). In conclusion, GSTD restored the mineralization ability of cells exposed to DEX treatment.

GSTD increases the expression of osteogenic gene markers in DEX-treated MC3T3-E1 cells. GCs at physiological levels are essential for the normal development of a wide range of tissues. 


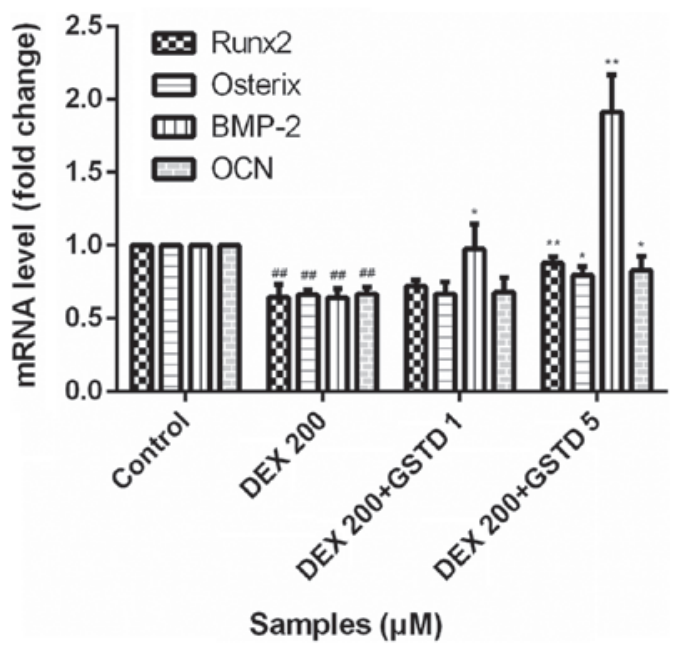

Figure 6. mRNA expression level of osteogenic genes in MC3T3-E1 cells exposed to dexamethasone (DEX) alone $(200 \mu \mathrm{M})$ or co-treated with gastrodin (GSTD) $(1$ and $5 \mu \mathrm{M})$ for $48 \mathrm{~h}$. The mRNA levels were analyzed using quantitative polymerase chain reaction, and the relative expression levels were calculated by comparison with the internal control glyceraldehyde 3-phosphate dehydrogenase. All experiments were performed in triplicate, and values are presented as mean \pm standard deviation. ${ }^{\# \#} \mathrm{P}<0.01$ vs. control; ${ }^{* * *} \mathrm{P}<0.01$ and ${ }^{*} \mathrm{P}<0.05$ vs. DEX $200 \mu \mathrm{M}$. BMP, bone morphogenetic protein; OCN, osteocalcin

DEX at physiological (low) concentrations is indispensable for osteoblastogenic differentiation and plays a pivotal role during maturity. To determine the effects of GSTD on the expression of osteogenic gene markers, the cells were pretreated with various concentrations of GSTD for $2 \mathrm{~h}$ followed by adding DEX at $200 \mu \mathrm{M}$ and incubating under osteogenic differentiation conditions for the next $48 \mathrm{~h}$. Osteogenic differentiation was assessed by using qPCR and measuring the mRNA expression levels of bone morphogenic protein-2 (BMP-2), Runx2, osterix and OCN. Based on qPCR, cells treated with $200 \mu \mathrm{M}$ DEX exhibited decreased mRNA expression levels of BMP-2, Runx2, OSX and OCN compared with the control, whereas these decreased expression levels were reversed in cells pretreated with GSTD, particularly demonstrated as changes in the expression of BMP-2 (Fig. 6), a cytokine most widely used to confer osteoinductivity, which accounts for osteogenic effect of osteoblasts to a great extent.

GSTD increases osteogenic transcription Runx2 and decreases adipogenic factor PPAR $\gamma$ and apoptosis-related caspase-3 protein expression levels via NRF2 pathway activation. To further investigate the mechanisms by which GSTD stimulates osteoblast differentiation under DEX treatment, western blotting was performed to examine the DEX- and GSTD-induced changes in Runx2, OCN, PPAR $\gamma$ isoform 2 and NRF2 pathway and its downstream effector protein NQO-1 and HO-1 expression (Fig. 7). Runx2, OCN, NQO-1 and HO-1 expression levels were markedly increased in MC3T3-E1 cells treated with different concentrations of GSTD $(1$ and $5 \mu \mathrm{M})$ compared with the cells treated with DEX alone, particularly in the $5 \mu \mathrm{M}$ group. On the contrary, PPAR $\gamma$ isoform 2 was downregulated, as was caspase-3. These findings indicate that GSTD promoted osteogenic rather than adipogenic differentiation and blocked apoptosis via activating NRF2 and its downstream effectors' HO-1 and NQO-1 protein expression.
Histological assessment of rat bone sections with $H \& E$ staining and BMD measurement indicate that GSTD alleviates bone loss induced by DEX. DEX at a high dose reduced bone generation and destroyed bone trabeculae in rats, leading to osteoporosis. Microanatomical changes to the bone microarchitecture were observed with H\&E staining (magnification, x100). The decreased density and number of trabeculae stained by eosin in the DEX group demonstrated severe bone loss compared with the control; however, GSTD alleviated this effect and restored bone mass (Fig. 8A), which was consistent with the results of the BMD measurement (Fig. 8B).

\section{Discussion}

Osteoporosis is a systemic skeletal disease characterized by decreased bone mass caused by a number of reasons, leading to weak and brittle bones (22). The clinical administration of GCs may result in iatrogenic osteoporosis, which disturbs osteoblast function, even induce apoptosis. GCs at physiological concentrations are vital for the natural development of bone, while high-dose administration leads to osteoporosis instead (23). Elucidation of the complex interplay between GCs and altered bone homeostasis is a subject of intense clinical interest. As DEX-induced bone loss is primarily mediated by osteoblast dysfunction, agents that act directly by either increasing osteoblast proliferation or inducing osteoblast differentiation are required to enhance bone formation. Several diverse natural pharmaceuticals exhibiting therapeutic efficacy through enhancement of bone formation, osteogenic differentiation and skeletal reconstruction, have been investigated $(23,24)$. The traditional Chinese herb Gastrodia elata contains an abundance of bioactive compounds of high pharmaceutical value and biomedical potential, among which, GSTD, a natural phenol extracted from Gastrodia elata, was recently demonstrated to exert potential protective effects against $\mathrm{H}_{2} \mathrm{O}_{2}$-induced injury in an oxidative hBMMSC model, and is considered as a potential candidate for promoting bone formation (8). However, the effects of GSTD on the proliferation and differentiation of osteoblastic cells under GC stimulation have yet to be elucidated. Therefore, in the present study, MC3T3-E1 cells were selected in order to investigate whether GSTD could antagonize DEX by stimulating cell proliferation and osteogenic differentiation, as well as preserving normal osteoblast function.

As previously stated, a decline in osteoblastic proliferation is a significant contributor to the pathogenesis of GIO (25), GCs inhibit osteoblast viability and proliferation, resulting in failure of osteogenesis. The CCK-8 assay was used to evaluate the effect of DEX and GSTD on cell viability and proliferation. A concentration of $200 \mu \mathrm{M}$ DEX was selected to construct the low-viability model using MC3T3-E1 cells. As shown in Fig. 2C, GSTD treatment at concentrations of 1 and $5 \mu \mathrm{M}$ restored cell viability in a dose-dependent manner. Therefore, these results indicated that low-dose GSTD, was not cytotoxic and promoted cell proliferation, reversing the effect of DEX.

Bone ALP is one of the phenotypic markers of osteoblasts, and plays a key role in osteogenesis. Its activity may directly reflect osteoblast activity and/or function. As previously mentioned, DEX inhibits initial osteoblast proliferation followed by decreasing ALP activity; thus, ALP activity was measured in DEX-treated osteoblasts following pretreatment 

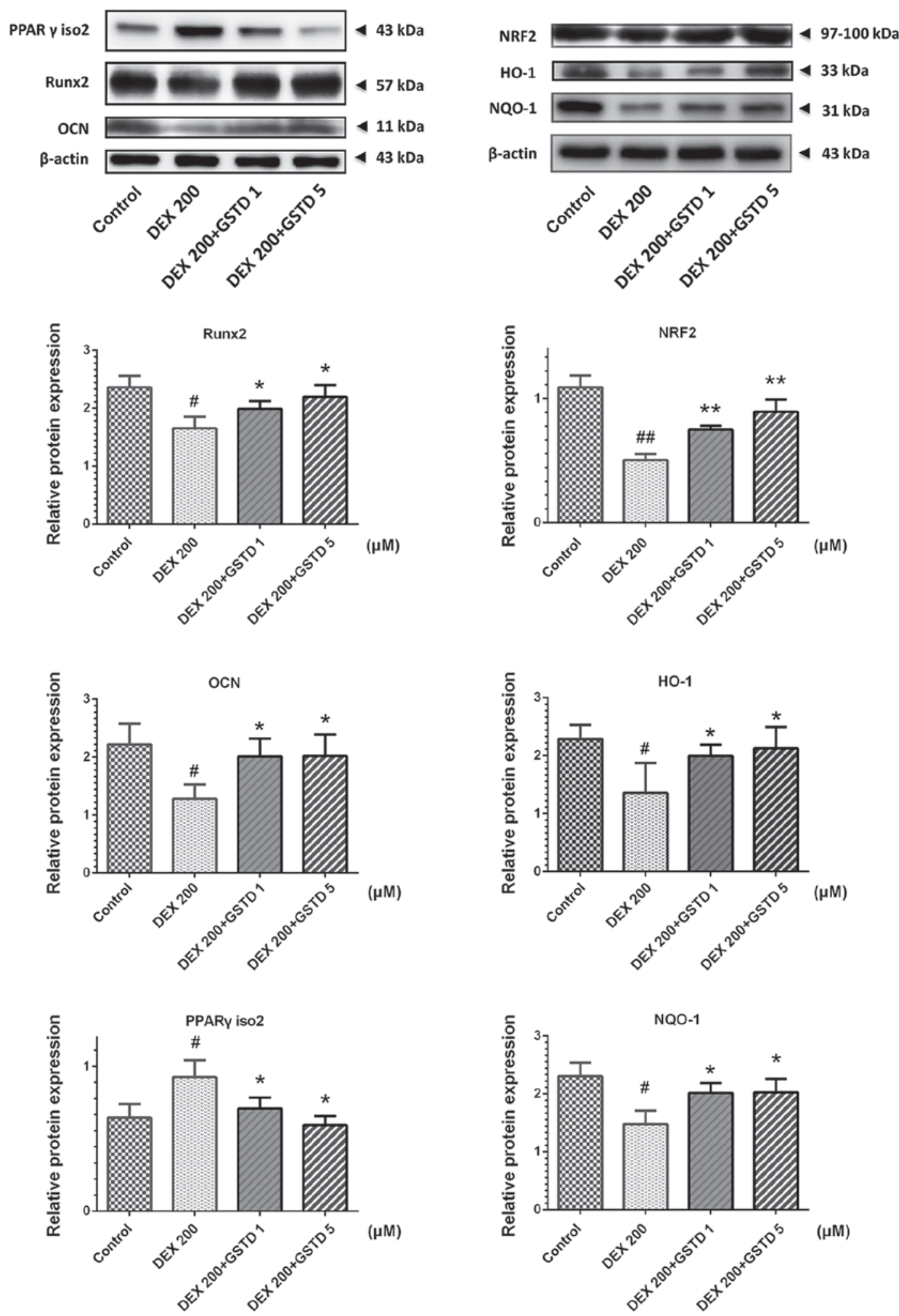

Figure 7. Gastrodin (GSTD) increased osteogenic transcription Runx2, decreased adipogenic factor peroxisome proliferator-activated receptor (PPAR) $\gamma$ isoform 2 and activated nuclear factor-like 2 (NRF2) pathway protein expression levels. Representative western blot analyses for total protein was analyzed by western blotting in dexamethasone (DEX)-treated MC3T3-E1 cells with or without GSTD pretreatment at different doses. The relative protein expression levels were measured using the fold-change in each protein relative to $\beta$-actin from the same sample. The data are expressed as the mean \pm standard deviation. ${ }^{\# \#} \mathrm{P}<0.01$ and ${ }^{\#} \mathrm{P}<0.05$ vs. control; ${ }^{*} \mathrm{P}<0.05$ and ${ }^{* *} \mathrm{P}<0.01$ vs. DEX. NQO-1, NAD(P)H:quinone oxidoreductase-1; HO-1, heme oxygenase-1; OCN, osteocalcin.

with GSTD at different concentrations of 1 and $5 \mu \mathrm{M}$. The results indicated that $200 \mu \mathrm{M}$ DEX decreased ALP activity by $\sim 30 \%$ compared with the control (Fig. 2D), while addition of GSTD restored ALP activity in a dose-dependent manner.
In addition, the changes in the expression levels of several key osteogenic genes, such as Runx2, BMP-2, OSX and OCN, were also investigated. The mRNA expression levels were assessed by qPCR. GSTD-incubated MC3T3-E1 cells exhib- 
A

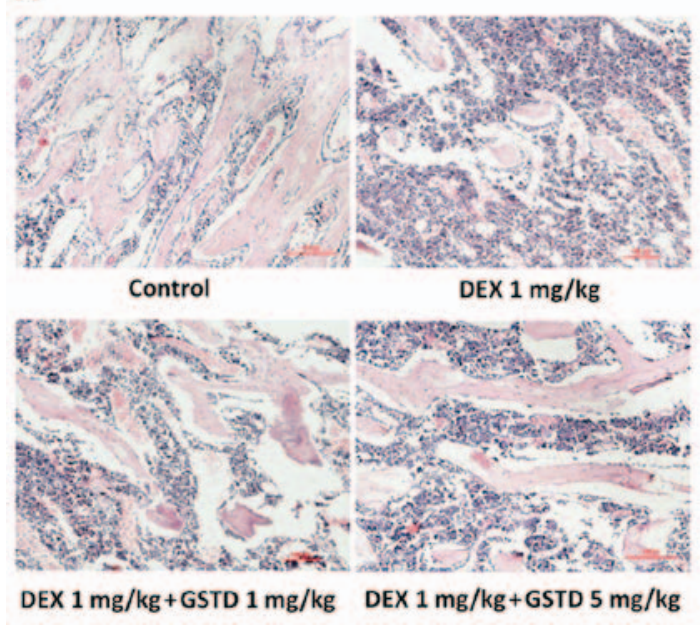

B

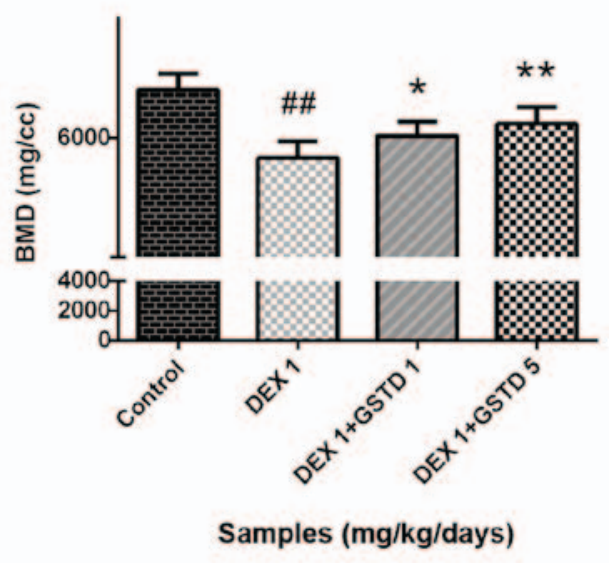

Figure. 8. (A) Effects of gastrodin (GSTD) on histological assessment of bone sections in rats (hematoxylin and eosin staining; magnification, x100). The areas stained red represent bone trabeculae. (B) Data from bone mineral density (BMD) measurement with dual X-ray absorptiometry in the femora of rats. The data are expressed as mean \pm standard deviation. ${ }^{\# \#} \mathrm{P}<0.01$ and ${ }^{\#} \mathrm{P}<0.05$ vs. control; ${ }^{*} \mathrm{P}<0.05$ and ${ }^{* *} \mathrm{P}<0.01$ vs. dexamethasone $(\mathrm{DEX})$.

ited higher mRNA expression of osteogenic transcription factors compared with cells treated with DEX alone, while, as a key signaling component of the TGF- $\beta$ superfamily, BMP-2 is indispensable for osteoblast proliferation and osteogenic differentiation, and may be the main contributor to this effect $(26,27)$; however, its mechanism of action requires further elucidation. Therefore, GSTD has the ability to promote osteogenesis against DEX treatment.

Runx2, also referred to as the core-binding factor $\alpha 1$ (Cbf $\alpha 1)$, is the most specific marker gene in the earliest stage of bone formation, and the expression of Runx 2 is a sign of osteogenic differentiation, osteogenesis and bone development. OCN is synthesized and secreted by osteoblasts, and it is more stable rather than easily affected by bone resorption factors, more accurately reflecting osteoblast activity. PPAR $\gamma$ is an adipogenic transcription factor regulating adipogenesis and is found as two isoforms, namely isoforms 1 and 2 (28), of which PPAR $\gamma$ isoform 2 directly regulates cell adipogenic differentiation when highly expressed. In the present study, treatment with DEX alone decreased Runx 2 expression and increased the level of PPAR $\gamma$ isoform 2 in MC3T3-E1 cells. As previously reported, GSTD is considered to effectively intervene with the effects of PPAR $\gamma$ in hypertensive rats (9). After pretreatment with GSTD, the cells were confirmed to exhibit reduced PPAR $\gamma$ expression and upregulated Runx2 and OCN expression, reflecting a reversal of the DEX effects, demonstrating that GSTD exerts a targeted effect against DEX-induced osteogenic differentiation dysfunction of osteoblasts. It has been reported that downregulating elevated reactive oxygen species levels in human mesenchymal stem cells is crucial for their proper differentiation to osteoblasts and osteogenic differentiation (29). NRF2 is an essential transcription factor that exerts cytoprotective effects through restoring the intracellular redox homeostasis, including downstream transcription effectors NQO-1and HO-1, by binding to ARE (30). DEX decreases NRF2 pathway protein expression possibly by inducing oxygen free radical generation; our study demonstrated that preincubation with GSTD effectively increased NRF2 and its downstream effectors' NQO-1 and HO-1 expression (Fig. 7A). This result indicates that oxidative stress accounts for the changes in the direction of MC3T3-E1 cell differentiation. In addition, GSTD promotes osteogenesis and maintains the balance of osteogenesis and adipogenesis by regulating NRF2 signaling pathway to a certain extent.

Apart from alterations in the differentiation characteristics at the molecular level, the process of bone formation also includes development and maturation of the extracellular matrix to mineralized deposits. Therefore, mineralization of extracellular matrix as $\mathrm{Ca}^{2+}$ deposits for mineralized nodule formation was assessed by Alizarin Red staining, which combines with $\mathrm{Ca}^{2+}$ ions. GSTD at the determined concentrations increased the formation of calcium nodules. These findings demonstrated that GSTD enhances the maturation process, upregulating osteoblast osteogenic differentiation in MC3T3-E1 cells. These results are in line with the findings at the molecular level mentioned above.

Mitochondria are the principal organelles considered to play a crucial role in osteoblasts, as they are involved in energy metabolism and calcium metabolism homeostasis, regulating cell survival and death, reflecting cellular function (31). Imbalance of mitochondrial dynamics may lead to cellular dysfunction and are the main cause of GC-induced bone metabolic disorders (32). It has been reported that GSTD may induce improvement in cellular mitochondrial function in several types of cells (8). We hypothesized that GSTD participates in repairing mitochondrial function, which is involved, at least in part, in the changes in cell function underlying GIO. Loss of MOMP is an early sign of cell apoptosis, representing mitochondrial dysfunction. Fluorescence shifting from red to green by JC-1 can be easily detected upon reduction of the membrane potential, and is also considered an indicator of DEX-induced early apoptosis. As shown in Fig. 4, $200 \mu \mathrm{M}$ DEX induced a 3 -folddecrease in MOMP compared with the control, while GSTD preincubation for $1 \mathrm{~h}$ increased the $\mathrm{red} / \mathrm{green}$ fluorescence ratio, indicating that GSTD mitigated 
the damage in mitochondrial function. In our study, the MOMP was measured using the JC-1 dye, and the findings suggested that GSTD attenuated the mitochondrial membrane injury in osteoblasts induced by DEX in vitro, indicating that GSTD improves DEX-induced dysfunction of M3T3-E1 cells by stabilizing MOMP.

It is universally acknowledged that DEX-induced osteoblast apoptosis significantly contributes to the development of GIO (33). Annexin V-FITC/PI staining-based flow cytometry analysis was applied to gain further insight into the effect of GSTD on DEX-induced osteoblast apoptosis. The results revealed that a large proportion of osteoblasts underwent apoptosis following exposure to $200 \mu \mathrm{M}$ DEX for $48 \mathrm{~h}$ (Fig. 3A). However, pretreatment with GSTD concentration-dependently attenuated DEX-induced apoptosis of osteoblasts. In particular, GSTD at a concentration of $5 \mu \mathrm{M}$ exerted a more potent effect on the decrease in apoptotic rate. These findings demonstrated that GSTD protects osteoblasts from DEX-induced apoptosis. Caspase-3 plays a key role in cell apoptosis, and its expression reflects apoptotic events, which is in line with the results of Annexin V-FITC/PI flow cytometry analysis. GSTD was able to reverse DEX-induced increase of caspase-3 expression (Fig. 3C and D) and protected osteoblasts from apoptosis.

In order to fully confirm that GSTD alleviates GIO, in the present study, rats were selected as the model of GIO, and the results demonstrated that GSTD obviously improved $\mathrm{DEX}$-induced osteoporosis in a rat model of $\mathrm{GIO}$ in vivo. $\mathrm{H} \& \mathrm{E}$ staining and BMD measurement were applied. The femur is the most vulnerable body region in terms of mechanical strength, and $\sim 40 \%$ of European women with atypical femoral fractures have received treatment with GCs at some point $(34,35)$. Hence, the femur was selected to evaluate the effects of GSTD on GIO. BMD, which is a useful index reflecting the status of bone metabolism, is broadly used to assess the changes in bone mass and predict the stiffness of the femur. GSTD relieved the erosive effects induced by DEX and increased the BMD, improving bone quality and strength.

GSTD was previously reported to protect ovariectomized rats against osteoporosis. However, the effect of GSTD on GIO remains unclear. It has been reported that GSTD may promote the activation of the NRF2 signaling pathway in other cells, playing a role in antioxidation and anti-inflammation. Furthermore, GSTD may also be used in hBMMSCs and promotes osteogenesis by reducing osteoclast differentiation. GSTD inhibits the key adipogenic differentiation factor PPAR $\gamma$, reducing the fat content in hypertensive model rats (37). In addition, GSTD was also found to improve mitochondrial function $(16,38)$. These interactions are closely associated with the mechanism of GIO. The present study mainly investigated the effects of GSTD on DEX-induced osteoblast dysfunction, and the findings were also validated in an animal osteoporotic model. Furthermore, GSTD targetedly modulated some essential factors in osteoblasts and attenuated the adverse effects of supra-physiological concentrations of DEX on osteoblasts.

As regards osteoclasts, experimental studies have established that GCs directly extend the lifespan of mature osteoclasts by delaying apoptotic signaling. Furthermore, the effects of GSTD on osteoclasts were well-documented in the study of Huang et al, who reported that GSTD suppressed osteoclastic differentiation in hBMMSCs and RAW264.7 cells, a type of preosteoclast cell (6). This study will hopefully provide new experimental data in the study of osteoclasts and help elucidate the mechanism of action of GSTD in DEX-induced osteoclasts in future studies, which may provide a new aproach to the development of new drugs for GIO treatment. Further research is required to provide more insight into DEX-induced osteoporosis and support the wider application of GSTD in clinical practice.

In conclusion, the present study demonstrated that GSTD enhanced osteogenic differentiation and bone formation under conditions of GC-induced dysfunction of MC3T3-E1 cells in vitro, through maintaining cell viability, stabilizing mitochondrial function, promoting osteogenic differentiation and decreasing cell apoptosis. However, further studies are required to fully elucidate the mechanism underlying GC-induced apoptosis in primary osteoblasts and the efficacy of GSTD in vivo for the treatment of GIO.

\section{Acknowledgements}

This study was sponsored by grants from the National Natural Science Foundation of China (no. 81370981) and the Outstanding Scientific Fund of Shengjing Hospital (MD31).

\section{Competing interests}

The authors declare that they have no competing interests.

\section{References}

1. Drake MT, Clarke BL and Lewiecki EM: The pathophysiology and treatment of osteoporosis. Clin Ther 37: 1837-1850, 2015

2. Seibel MJ, Cooper MS and Zhou H: Glucocorticoid-induced osteoporosis: Mechanisms, management, and future perspectives. Lancet Diabetes Endocrinol 1: 59-70, 2013.

3. Frenkel B, White W and Tuckermann J: Glucocorticoid-induced osteoporosis. Adv Exp Med Biol 872: 179-215, 2015.

4. Henneicke H, Gasparini SJ, Brennan-Speranza TC, Zhou H and Seibel MJ: Glucocorticoids and bone: Local effects and systemic implications. Trends Endocrinol Metab 25: 197-211, 2014.

5. Buehring $\mathrm{B}$, Viswanathan R, Binkley $\mathrm{N}$ and Busse $\mathrm{W}$ : Glucocorticoid-induced osteoporosis: An update on effects and management. J Allergy Clin Immunol 132: 1019-1030, 2013.

6. Huang Q, Shi J, Gao B, Zhang HY, Fan J, Li XJ, Fan JZ, Han YH, Zhang JK, Yang L, et al: Gastrodin: An ancient Chinese herbal medicine as a source for anti-osteoporosis agents via reducing reactive oxygen species. Bone 73: 132-144, 2015.

7. Zheng H, Yang E, Peng H, Li J, Chen S, Zhou J, Fang H, Qiu B and Wang Z: Gastrodin prevents steroid-induced osteonecrosis of the femoral head in rats by anti-apoptosis. Chin Med J (Engl) 127: 3926-3931, 2014

8. Qu LL, Yu B, Li Z, Jiang WX, Jiang JD and Kong WJ: Gastrodin ameliorates oxidative stress and proinflammatory response in nonalcoholic fatty liver disease through the AMPK/Nrf2 pathway. Phytother Res 30: 402-411, 2016.

9. Peng Z, Wang S, Chen G, Cai M, Liu R, Deng J, Liu J, Zhang T, Tan Q and Hai C: Gastrodin alleviates cerebral ischemic damage in mice by improving anti-oxidant and anti-inflammation activities and inhibiting apoptosis pathway. Neurochem Res 40: 661-673, 2015.

10. Sun YX, Xu AH, Yang Y and Li J: Role of Nrf2 in bone metabolism. J Biomed Sci 22: 101, 2015.

11. Loboda A, Damulewicz M, Pyza E, Jozkowicz A and Dulak J: Role of Nrf2/HO-1 system in development, oxidative stress response and diseases: An evolutionarily conserved mechanism. Cell Mol Life Sci 73: 3221-3247, 2016.

12. Sun YX, Li L, Corry KA, Zhang P, Yang Y, Himes E, Mihuti CL, Nelson C, Dai G and Li J: Deletion of Nrf2 reduces skeletal mechanical properties and decreases load-driven bone formation. Bone 74: 1-9, 2015. 
13. Lin H, Wei B, Li G, Zheng J, Sun J, Chu J, Zeng R and Niu Y: Sulforaphane reverses glucocorticoid-induced apoptosis in osteoblastic cells through regulation of the Nrf2 pathway. Drug Des Devel Ther 8: 973-982, 2014.

14. Pellegrini GG, Morales CC, Wallace TC, Plotkin LI and Bellido T: Avenanthramides prevent osteoblast and osteocyte apoptosis and induce osteoclast apoptosis in vitro in an Nrf2-independent manner. Nutrients 8: 423, 2016.

15. Choi EM: Magnolol protects osteoblastic MC3T3-E1 cells against antimycin A-induced cytotoxicity through activation of mitochondrial function. Inflammation 35: 1204-1212, 2012.

16. Yuan Z, Li Q, Luo S, Liu Z, Luo D, Zhang B, Zhang D, Rao P and Xiao J: PPAR $\gamma$ and Wnt signaling in adipogenic and osteogenic differentiation of mesenchymal stem cells. Curr Stem Cell Res Ther 11: 216-225, 2016.

17. Rauch A, Seitz S, Baschant U, Schilling AF, Illing A, Stride B, Kirilov M, Mandic V, Takacz A, Schmidt-Ullrich R, et al: Glucocorticoids suppress bone formation by attenuating osteoblast differentiation via the monomeric glucocorticoid receptor. Cell Metab 11: 517-531, 2010.

18. Hartmann K, Koenen M, Schauer S, Wittig-Blaich S, Ahmad M, Baschant U and Tuckermann JP: Molecular actions of glucocorticoids in cartilage and bone during health, disease, and steroid therapy. Physiol Rev 96: 409-447, 2016.

19. Yu S, Yerges-Armstrong LM, Chu Y, Zmuda JM and Zhang Y: E2F1 effects on osteoblast differentiation and mineralization are mediated through upregulation of frizzled-1. Bone 56: 234-241, 2013.

20. Chen Z, Xue J, Shen T, Ba G, Yu D and Fu Q: Curcumin alleviates glucocorticoid-induced osteoporosis by protecting osteoblasts from apoptosis in vivo and in vitro. Clin Exp Pharmacol Physiol 43: 268-276, 2016.

21. Fang J, Yamaza H, Uchiumi T, Hoshino Y, Masuda K, Hirofuji Y, Wagener FA, Kang D and Nonaka K: Dihydroorotate dehydrogenase depletion hampers mitochondrial function and osteogenic differentiation in osteoblasts. Eur J Oral Sci 124: 241-245, 2016.

22. Chan CK, Mason A, Cooper C and Dennison E: Novel advances in the treatment of osteoporosis. Br Med Bull 119: 129-142, 2016.

23. Kalak R, Zhou H, Street J, Day RE, Modzelewski JR, Spies CM, Liu PY, Li G, Dunstan CR and Seibel MJ: Endogenous glucocorticoid signalling in osteoblasts is necessary to maintain normal bone structure in mice. Bone 45: 61-67, 2009.

24. Chen Z, Xue J, Shen T, Mu S and Fu Q: Curcumin alleviates glucocorticoid-induced osteoporosis through the regulation of the Wnt signaling pathway. Int J Mol Med 37: 329-338, 2016.

25. Kim J, Lee H, Kang KS, Chun KH and Hwang GS: Protective effect of Korean Red Ginseng against glucocorticoid-induced osteoporosis in vitro and in vivo. J Ginseng Res 39: 46-53, 2015.

26. Liang W, Lin M, Li X, Li C, Gao B, Gan H, Yang Z, Lin X, Liao L and Yang M: Icariin promotes bone formation via the BMP-2/Smad4 signal transduction pathway in the hFOB 1.19 human osteoblastic cell line. Int J Mol Med 30: 889-895, 2012.
27. Cao H, Ke Y, Zhang Y, Zhang CJ, Qian W and Zhang GL: Icariin stimulates MC3T3-E1 cell proliferation and differentiation through upregulation of bone morphogenetic protein-2. Int J Mol Med 29: 435-439, 2012.

28. Zhuang H, Zhang X, Zhu C, Tang X, Yu F, Shang GW and Cai X Molecular mechanisms of PPAR- $\gamma$ governing MSC osteogenic and adipogenic differentiation. Curr Stem Cell Res Ther 11: 255-264, 2016.

29. Gómez-Puerto MC, Verhagen LP, Braat AK, Lam EW, Coffer PJ and Lorenowicz MJ: Activation of autophagy by FOXO3 regulates redox homeostasis during osteogenic differentiation. Autophagy 12: 1804-1816, 2016.

30. Choi EM, Suh KS, Kim YJ, Hong SM, Park SY and Chon S: Glabridin alleviates the toxic effects of methylglyoxal on osteoblastic MC3T3-E1 cells by increasing expression of the glyoxalase system and $\mathrm{Nrf} 2 / \mathrm{HO}-1$ signaling and protecting mitochondrial function. J Agric Food Chem 64: 226-235, 2016.

31. Angelova PR and Abramov AY: Functional role of mitochondrial reactive oxygen species in physiology. Free Radic Biol Med 100: 81-85, 2016 .

32. Zhen YF, Wang GD, Zhu LQ, Tan SP, Zhang FY, Zhou XZ and Wang XD: P53 dependent mitochondrial permeability transition pore opening is required for dexamethasone-induced death of osteoblasts. J Cell Physiol 229: 1475-1483, 2014.

33. O'Brien CA, Jia D, Plotkin LI, Bellido T, Powers CC, Stewart SA, Manolagas SC and Weinstein RS: Glucocorticoids act directly on osteoblasts and osteocytes to induce their apoptosis and reduce bone formation and strength. Endocrinology 145: 1835-1841, 2004.

34. de Vries F, Pouwels S, Lammers JW, Leufkens HG, Bracke M, Cooper C and van Staa TP: Use of inhaled and oral glucocorticoids, severity of inflammatory disease and risk of hip/ femur fracture: A population-based case-control study. J Intern Med 261: 170-177, 2007.

35. Giusti A, Hamdy NA and Papapoulos SE: Atypical fractures of the femur and bisphosphonate therapy: A systematic review of case/case series studies. Bone 47: 169-180, 2010.

36. Zhang ZC, Su G, Li J, Wu H and Xie XD: Two new neuroprotective phenolic compounds from Gastrodia elata. J Asian Nat Prod Res 15: 619-623, 2013

37. Liu W, Wang L, Yu J, Asare PF and Zhao YQ: GSTD reduces blood pressure by intervening with RAAS and PPAR $\gamma$ in SHRs. Evid Based Complement Alternat Med 2015: 828427, 2015.

38. Wang XL, Xing GH, Hong B, Li XM, Zou Y, Zhang XJ and Dong MX: Gastrodin prevents motor deficits and oxidative stress in the MPTP mouse model of Parkinson's disease: Involvement of ERK1/2-Nrf2 signaling pathway. Life Sci 114: 77-85, 2014.

This work is licensed under a Creative Commons Attribution-NonCommercial-NoDerivatives 4.0 International (CC BY-NC-ND 4.0) License. 